\title{
Short communication: Antimicrobial resistance and virulence characterization of methicillin-resistant staphylococci isolates from bovine mastitis cases in Portugal
}

\author{
R. Seixas, J. P. Santos, R. Bexiga, C. L. Vilela, and M. Oliveira ${ }^{1}$ \\ Immunology and Microbiology Laboratory, Interdisciplinary Centre of Research in Animal Health (CIISA)/Faculdade de Medicina Veterinária \\ da Universidade de Lisboa, Avenida da Universidade Técnica, 1300-477 Lisboa, Portugal
}

\section{ABSTRACT}

Methicillin-resistant staphylococci (MRS) have already been reported as mastitis agents. Such bacterial species are a public health concern, and the characterization of their antimicrobial resistance and virulence profile is important to better control their dissemination. The present work evaluated the distribution of methicillin-resistance among 204 staphylococci from clinical $(\mathrm{n}=50)$ and subclinical $(\mathrm{n}=154)$ bovine mastitis. The presence of the mecA gene was determined by PCR. Phenotypic expression of coagulase, DNase, lipase, gelatinase, hemolytic enzymes, and biofilm production was evaluated. The presence of biofilm-related genes, $i c a \mathrm{~A}$, $i c a \mathrm{D}$, and bap, was also determined. Antimicrobial resistance patterns for aminoglycosides, lincosamides, macrolides, fluoroquinolones, sulphonamides, tetracyclines, and fusidic acid were determined. Nineteen (9.3\%) isolates were identified as MRS, and the presence of mecA in these isolates was confirmed by PCR. Virulence factors evaluation revealed that gelatinase was the most frequently detected $(94.7 \%)$, followed by hemolysins $(73.7 \%)$ and lipase $(68.4 \%) ; 84.2 \%$ of the MRS isolates produced biofilm and icaA and $i c a \mathrm{D}$ were detected in almost half of the MRS isolates $(52.6 \%)$, but all were bap-negative. Resistance against other antimicrobial agents ranged from 0 (fusidic acid, ciprofloxacin, norfloxacin, enrofloxacin) to $100 \%$ (nalidixic acid). Resistance to nalidixic acid and nalidixic acid-tetracycline were the most common antimicrobial resistance profiles $(31.6 \%)$. This study confirms that despite the low prevalence of MRS, isolates frequently express other virulence traits, especially biofilm, that may represent a serious challenge to clinicians.

Key words: staphylococci, biofilm, methicillin resistance, bovine mastitis

Received June 12, 2013.

Accepted October 4, 2013.

${ }^{1}$ Corresponding author: moliveira@fmv.utl.pt

\section{Short Communication}

In dairy farms, mastitis remains an important disease with high economic effect. Although many microorganisms have been implicated with this disease, Staphylococcus aureus has been described as a major pathogen responsible for bovine mastitis; whereas Staphylococcus epidermidis is being increasingly isolated from clinical and subclinical mastitis (Pyörälä and Taponen, 2009). Since the introduction of methicillin in clinical practice in the 1960s, the increasing resistance of staphylococci to $\beta$-lactamic antimicrobial compounds has become a major problem. The first methicillin-resistant Staphylococcus aureus (MRSA) from animal origin was isolated in 1975 from milk samples obtained from cows with mastitis (Kawano et al., 1996). More recently, MRSA and methicillinresistant Staph. epidermidis are frequently isolated from cattle (Walther and Perreten, 2007). Nevertheless, infection due to these bacterial species is rare, and MRSA reports related with bovine mastitis are scarce (Juhász-Kaszanyitzky et al., 2007).

However, methicillin-resistant staphylococci (MRS) may be a serious concern for dairy production, as they can express a wide range of virulence traits including surface proteins, exoenzymes, and extracellular toxins, which may strongly influence infection prognosis (Gordon and Lowy, 2008). Biofilm, a recognized virulence factor in staphylococci, has been proposed as a significant element in the persistence of IMI (Tremblay et al., 2013). The intercellular adhesion (ica) locus is responsible for the production of a polysaccharide intercellular adhesin and a capsular polysaccharide, 2 major components of staphylococci biofilms. Among the ica genes, icaA and icaD play a key role in biofilm formation (Arciola et al., 2001). Staphylococcus aureus strains harboring the biofilm-associated protein (bap) gene are found to be strong biofilm producers (Vancraeynest et al., 2004).

The goal of this study was to characterize the antimicrobial resistance profile and virulence traits' distribution among MRS isolates, from a collection of bovine 
clinical and subclinical mastitis staphylococci isolates ( $\mathrm{n}=204)$ gathered from 2004 to 2010 belonging to the Immunology and Microbiology Laboratory of the Faculty of Veterinary Medicine, University of Lisbon. Samples were collected from dairy cows belonging to commercial dairy farms located in the Ribatejo-Oeste area of Portugal. Collection of milk samples of bovine subclinical and clinical mastitis and staphylococci isolation were performed according to the National Mastitis Council protocols (National Mastitis Council, 1987). Milk samples of approximately $5 \mathrm{~mL}$ were collected aseptically. A volume of $10 \mu \mathrm{L}$ was plated on Columbia agar supplemented with $5 \%$ sheep blood (bioMérieux, Marcy l'Etoile, France) and isolation was performed when colonies of similar morphology were in a number higher that $500 \mathrm{cfu} / \mathrm{mL}$.

Screening of methicillin resistance was performed by the disc diffusion method using oxacillin discs $(1 \mu \mathrm{g}$; Oxoid, Basingstoke, United Kingdom), according to Clinical and Laboratory Standards Institute (CLSI) guidelines (CLSI, 2008). Results were confirmed by the MIC method using oxacillin $(0.25-4 \mu \mathrm{g} / \mathrm{mL}$, SigmaAldrich, St. Louis, MO), as recommended by CLSI guidelines. Test performance was monitored using Staph. aureus ATCC 25923 and performed in duplicate.

Confirmation of methicillin resistance was performed by conventional PCR targeting the mecA gene, using the protocol described in Pereira et al. (2010). The MRSA control strain was kindly provided by Constança Pomba (Faculdade de Medicina Veterinária da Universidade de Lisboa, Portugal). The DNA template for PCR amplification was obtained from a single colony of an overnight culture $\left(18 \mathrm{~h}, 37^{\circ} \mathrm{C}\right)$, using the guanidine thiocyanate method (Merck, Darmstadt, Germany; Pitcher et al., 1989).

Identification of bacterial species was confirmed by PCR according to Pereira et al. (2010) for Staph. aureus and Staph. epidermidis. Other staphylococci were confirmed for genus using the protocol described by Martineau et al. (1998). Staphylococcus epidermidis ATCC 12228 and Staph. aureus CECT 4513 were used as PCR amplification controls.

Antimicrobial coresistance profiles were determined using the disc diffusion method according to CLSI guidelines, for aminoglycosides [gentamicin $(10 \mu \mathrm{g})$, kanamycin $(30 \mu \mathrm{g})$, neomycin (10 $\mu \mathrm{g}$; Oxoid), streptomycin $(10 \mu \mathrm{g}$; Oxoid), tobramycin $(10 \mu \mathrm{g})]$, lincosamides [clindamycin $(2 \mu \mathrm{g})$ ], macrolides [erythromycin $(15 \mu \mathrm{g})$ ], fluoroquinolones [ciprofloxacin $(5 \mu \mathrm{g})$, enrofloxacin $(5 \mu \mathrm{g})$, nalidixic acid $(30 \mu \mathrm{g})$, norfloxacin (10 $\mu \mathrm{g})$ ], sulphonamides [sulphamethoxazole-trimethoprim $(25 \mu \mathrm{g})]$, tetracyclines [tetracycline $(30 \mu \mathrm{g})]$, and steroid antibacterials [fusidic acid $(10 \mu \mathrm{g})$ ]. All discs were purchased from Oxoid; Staph. aureus ATCC 25923 was used as a control for test performance.

All isolates identified as MRS were characterized for virulence traits by phenotypic methods. This characterization included the determination of coagulase activity (Liofilchem, Roseto degli Abruzzi, Italy; Sperber and Tatini, 1975) and DNase (Liofilchem; Weckman and Catlin, 1957), gelatinase (Liofilchem; Semedo et al., 2003), lipase (Becton Dickinson, Pont de Claix, France), and production of hemolysins (bioMérieux; Pereira et al., 2009). Staphylococcus aureus CECT 4513, Staph. epidermidis ATCC 12228, and Staph. aureus ATCC 25923 were used as controls for good quality standards.

Biofilm-forming ability by MRS isolates was evaluated by both phenotypic and genotypic methods. Phenotypic expression was evaluated using a fluorescence in situ hybridization (FISH) protocol (Oliveira et al., 2007). Genes involved in biofilm production, including $i c a \mathrm{~A}$, ica $\mathrm{D}$, and bap, were identified by conventional PCR (Vasudevan et al., 2003; Vancraeynest et al., 2004). Staphylococcus epidermidis ATCC 35984 (RP62A) and Staph. epidermidis ATCC 12228 were used as biofilm and nonbiofilm producer control strains, respectively.

Methicillin resistance screening by oxacillin disc diffusion detected 19 (9.3\%) MRS, identified as Staph. epidermidis ( $\mathrm{n}=16 ; 84.2 \%)$, Staphylococcus haemolyticus $(\mathrm{n}=1 ; 5.3 \%)$, Staphylococcus simulans $(\mathrm{n}=1 ; 5.3 \%)$ and Staphylococcus chromogenes $(\mathrm{n}=1 ; 5.3 \%)$. These isolates were obtained from cows with bovine clinical (n $=3 ; 15.8 \%)$ and subclinical mastitis $(\mathrm{n}=16 ; 84.2 \%)$. Oxacillin MIC results were interpreted according to CLSI guidelines M31-A3 (CLSI, 2008) breakpoints and showed that most isolates $(78.9 \%)$ presented resistant MIC results $\geq 4 \mu \mathrm{g} / \mathrm{mL}$. Only 3 isolates showed different results between disc diffusion and MIC determination. Methicillin resistance was confirmed by mecA PCR amplification in all 19 isolates.

Resistance to other antimicrobial agents ranged from 0 (fusidic acid and ciprofloxacin, norfloxacin enrofloxacin) to $100 \%$ (nalidixic acid). None of the isolates was susceptible to all antimicrobials tested, whereas the vast majority (16 isolates) showed a multiresistance profile. The most common antimicrobial resistance profiles were resistance to nalidixic acid and nalidixic acid-tetracycline $(31.6 \%$; Table 1$)$.

Virulence traits characterization revealed that no isolate was coagulase-positive, 14 isolates produced hemolysins (73.7\%), 2 produced DNase (10.5\%), 18 were gelatinase-positive (94.7\%), and 13 were lipase-positive (68.4\%). Biofilm production revealed that $84.2 \%$ of the isolates $(\mathrm{n}=16)$ were able to produce biofilm after $24 \mathrm{~h}$ of incubation. The PCR amplifications revealed 3 isolates were only positive for ica $\mathrm{D}(15.8 \%)$ and 10 
Table 1. Antimicrobial resistance and virulence profiles of methicillin-resistant staphylococci isolates from bovine clinical and subclinical mastitis

\begin{tabular}{|c|c|c|c|c|c|}
\hline $\begin{array}{l}\text { Isolate } \\
\text { identification } \\
\text { code }\end{array}$ & Identification & MIC & $\begin{array}{l}\text { Antimicrobial coresistance } \\
\text { patterns }^{1}\end{array}$ & $\begin{array}{l}\text { Virulence } \\
\text { phenotype }^{2}\end{array}$ & $\begin{array}{l}\text { Biofilm phenotype } \\
\text { and genes } \\
(i c a \mathrm{~A}, i c a \mathrm{D}, b a p)^{3}\end{array}$ \\
\hline MC 13 & Staphylococcus epidermidis & $\geq 4$ & NA & Lip & Negative \\
\hline $102-2 \mathrm{Q} 979 \mathrm{RH}$ & Staphylococcus epidermidis & $\geq 0.5$ & NA & Lip; Gel & Positive \\
\hline MC 11 & Staphylococcus epidermidis & $\geq 4$ & NA; E; K; CN; TOB & Lip; Hae $\beta$; Gel & Negative \\
\hline 9336 & Staphylococcus epidermidis & $\geq 4$ & NA; E; K; TE; CN; TOB; N; S & Hae $\beta ;$ Gel & Positive; $i c a \mathrm{~A}, i c a \mathrm{D}$ \\
\hline 7706 & Staphylococcus epidermidis & $\geq 4$ & $\begin{array}{l}\text { NA; E; K; TE; CN; TOB; N; } \\
\text { S; DA }\end{array}$ & Hae $\beta$; Gel & Positive; $i c a \mathrm{~A}, i c a \mathrm{D}$ \\
\hline 162 & Staphylococcus epidermidis & 1 & NA; E; K; TE; TOB; N; S; DA & Hae $\alpha$; Gel & Positive; $i c a \mathrm{~A}, i c a \mathrm{D}$ \\
\hline $100-3 \mathrm{Q} 1045 \mathrm{LF} 1$ & Staphylococcus simulans & 2 & NA; E; S; DA & Lip; Gel & Positive \\
\hline D9326 & Staphylococcus epidermidis & $\geq 4$ & NA; K; CN; TOB; N; S; SXT & Gel & Positive; $i c a \mathrm{~A}, i c a \mathrm{D}$ \\
\hline 7125 & Staphylococcus epidermidis & 4 & NA; K; TE; CN; TOB; N; S & Hae $\beta$; Gel & Positive; $i c a \mathrm{~A}, i c a \mathrm{D}$ \\
\hline F7029 & Staphylococcus epidermidis & 4 & NA; K; TE; CN; TOB; S; SXT & Lip; Hae $\beta$; Gel & Positive; $i c a \mathrm{~A}, i c a \mathrm{D}$ \\
\hline $109-10 \mathrm{Q} 1397 \mathrm{LH} 1$ & Staphylococcus chromogenes & $\overline{\geq} 4$ & $\mathrm{NA} ; \mathrm{TE}$ & Lip; Gel & Positive \\
\hline 7059 & Staphylococcus epidermidis & $\geq 4$ & NA; TE; S; SXT & Lip; Hae $\beta$; Gel & Positive; $i c a \mathrm{~A}, i c a \mathrm{D}$ \\
\hline
\end{tabular}

${ }^{1} \mathrm{NA}=$ nalidixic acid; $\mathrm{E}=$ erythromycin; $\mathrm{K}=$ kanamycin; $\mathrm{TE}=$ tetracycline; $\mathrm{CN}=$ gentamicin; $\mathrm{TOB}=$ tobramycin; $\mathrm{N}=$ neomycin; $\mathrm{S}=$ streptomycin; DA = clindamycin; SXT = sulphamethoxazole-trimethoprim.

${ }^{2} \mathrm{Lip}=$ lipase; Hae $=$ hemolysins $(\alpha$ and $\beta) ;$ Gel $=$ gelatinase.

${ }^{3} i c a=$ intercellular adhesion; bap $=$ biofilm-associated protein.

isolates were both $i c a \mathrm{~A}$ and $i c a \mathrm{D}$ positive (52.6\%); all isolates were bap-negative.

In the current study, 204 staphylococci isolates were screened for methicillin resistance by oxacillin disc diffusion method according to CLSI guidelines. Afterward, a collection of 19 methicillin-resistant isolates were selected for further virulence characterization, with Staph. epidermidis being the most predominant species (84.2\%). Other studies (Piessens et al., 2011) regard Staph. epidermidis as an emerging pathogen frequently isolated form clinical and subclinical mastitis, and methicillin-resistant Staph. epidermidis have already been reported among mastitis isolates (Walther and Perreten, 2007).

Disc diffusion and MIC determination using oxacillin are 2 standard methods to detect resistance to methicillin. However, these 2 techniques are not always in accordance, as previously reported (Sakoulas and Moellering, 2008). In this case, 2 isolates presented consistently resistant results in disk diffusion, showed susceptible values in MIC determination, and were mecA negative. This could be explained by the production of $\beta$-lactamase (Ghoshal et al., 2004). It was also observed that one isolate, identified as Staph. haemolyticus, was resistant to oxacillin and $m e c \mathrm{~A}$ positive but susceptible to methicillin by MIC determination, which could be due to the lack of induction of oxacillin resistance (Ghoshal et al., 2004).
The extensive use or misuse of broad-spectrum antimicrobials is one of several risk factors that can lead to the emergence of multiresistant staphylococci (Virdis et al., 2010). In that study, high levels of resistance in the MRS isolates were observed for nalidixic acid $(100 \%)$, tetracycline $(57.9 \%)$, and aminoglycosides, such as streptomycin $(52.6 \%)$, kanamycin, and tobramycin (47.4\%). Lack of resistance was detected for fusidic acid and fluoroquinolones, including ciprofloxacin, enrofloxacin, and norfloxacin, with all isolates being susceptible to these compounds. Despite these antimicrobials being active in MRS, they are not good therapeutic options for the treatment of bovine mastitis because fusidic acid is unavailable in veterinary medicine and fluoroquinolones are mostly used for systemic treatment of gram-negative mastitis infection (Pyörälä, 2006). However, despite the high levels of susceptibility in in vitro tests, the lack of studies of fluoroquinolones in bovine mastitis infections by staphylococci, the broad spectrum and the higher cost of these antimicrobials, make these agents a poor therapeutic option.

$\beta$-Lactams are also restricted, as they are not clinically effective against MRS (Clinical and Laboratory Standards Institute, 2008). Virulence factors are widely distributed among staphylococci and are related with bacterial pathogenicity, as they may enhance tissue colonization and destruction and may also contribute to bacterial evasion from host defenses (Turkyilmaz and 
Kaya, 2006). In our study, all staphylococci expressed at least one of the virulence traits tested. Almost all isolates were gelatinase-positive, and a vast majority produced hemolysins and lipases; these results are in agreement with other authors (Turkyilmaz and Kaya, 2006). Although MRS strains are not necessarily more virulent than methicillin-susceptible staphylococci, MRS strains associated with degradative and invasive virulence factors, such as gelatinases and lipases, represent a more complex challenge to clinicians with potentially greater costs and more limited treatment options (Gordon and Lowy, 2008).

In recent years, biofilm-forming ability has been increasingly recognized as an important virulence trait in staphylococci (Tremblay et al., 2013). In this study, the FISH protocol showed that $84.2 \%$ of the isolates were biofilm producers. Other studies reported similar or higher percentages of biofilm-producing staphylococci (Arciola et al., 2001; Vasudevan et al., 2003; Oliveira et al., 2006).

It was observed that some isolates were biofilmpositive on FISH and icaA- and icaD-negative. Strains with a biofilm-positive phenotype but negative for the presence of these genes can be explained by the existence of other mechanisms of biofilm production, which has already been demonstrated for staphylococci (Fitzpatrick et al., 2005). The percentage of icaA and $i c a \mathrm{D}$ positive isolates was lower in comparison with others reports (Vasudevan et al., 2003; Dhanawade et al., 2010). Nevertheless, the fact that $68.4 \%$ of the isolates were $i c a$-positive reveals an enhanced ability of these clinical isolates to form biofilm compared with saprophytic strains, usually negative for both $i c a \mathrm{~A}$ and $i c a \mathrm{D}$ genes, not being able to produce biofilm (Arciola et al., 2001; Vasudevan et al., 2003). The presence of icaA or the combination of multiple genes is associated with a greater ability to produce biofilm (Tremblay et al., 2013). This result, allied to fact that all strains tested were methicillin-resistant, may pose an important challenge to veterinary practitioners who must deal with chronic intramammary relapsing infections with limited antimicrobial therapeutic options. Regarding PCR amplification of bap gene, all MRS isolates were negative. This is in accordance with previous studies, which report low prevalence of this gene among staphylococci isolates (Vancraeynest et al., 2004; Vautor et al., 2008).

In this study, isolation of MRS from bovine mastitis was low (9.3\%). Antimicrobial resistance of these isolates showed limited therapeutic options available, which re-enforces the need for monitoring methicillin resistance and coresistance profiles among staphylococcal populations isolated from bovine infections to prevent and control the spread of these strains.

\section{ACKNOWLEDGMENTS}

We dedicate this work to Cristina Lobo Vilela (1958-2013), a major contributor to the research presented in this manuscript. This work was supported by CIISA (Interdisciplinary Centre of Research in Animal Health). Rui Seixas holds a BD fellowship (SFRH/BD/75836/2011) from the Foundation for Science and Technology, Lisbon, Portugal. M. Oliveira is a researcher from the program "Ciência 2007" from the Foundation for Science and Technology, Portugal.

\section{REFERENCES}

Arciola, C. R., L. Baldassarri, and L. Montanaro. 2001. Presence of $i c a \mathrm{~A}$ and $i c a \mathrm{D}$ genes and slime production in a collection of staphylococcal strains from catheter-associated infections. J. Clin. Microbiol. 39:2151-2156.

CLSI (Clinical and Laboratory Standards Institute). 2008. Performance standards for antimicrobial disk and dilution susceptibility tests for bacteria isolated from animals. Approved Standard document M31-A3. 3rd ed. CLSI, Wayne, PA.

Dhanawade, N. B., D. R. Kalorey, R. Srinivasan, S. B. Barbuddhe, and N. V. Kurkure. 2010. Detection of intercellular adhesion genes and biofilm production in Staphylococcus aureus isolated from bovine subclinical mastitis. Vet. Res. Commun. 34:81-89.

Fitzpatrick, F., H. Humphreys, and J. P. O'Gara. 2005. Evidence for icaADBC-independent biofilm development mechanism in methicillin-resistant Staphylococcus aureus clinical isolates. J. Clin. Microbiol. 43:1973-1976.

Ghoshal, U., K. N. Prasad, M. Singh, D. P. Tiwari, and A. Ayyagari. 2004. A comparative evaluation of phenotypic and molecular methods for the detection of oxacillin resistance in coagulase-negative staphylococci. J. Infect. Chemother. 10:86-89.

Gordon, R. J., and F. D. Lowy. 2008. Pathogenesis of methicillin-resistant Staphylococcus aureus infection. Clin. Infect. Dis. 46:S350359 .

Juhász-Kaszanyitzky, E., S. Jánosi, P. Somogyi, A. Dán, L. van der Graaf-van Bloois, E. van Duijkeren, and J. A. Wagenaar. 2007. MRSA transmission between cows and humans. Emerging Infect. Dis. 13:630-632.

Kawano, J., A. Shimizy, S. Saitoh, T. Saito, M. Yagi, and R. Okamoto. 1996. Isolation of methicillin-resistant coagulase-negative staphylococci from chickens. J. Clin. Microbiol. 34:2072-2077.

Martineau, F., F. Picard, P. Roy, M. Ouellette, and M. Bergeron. 1998. Species-specific and ubiquitous-DNA-based assays for rapid identification of Staphylococcus aureus. J. Clin. Microbiol. 36:618-623.

National Mastitis Council. 1987. Laboratory and Field Handbook on Bovine Mastitis. Natl. Mastitis Counc., Verona, WI.

Oliveira, M., R. Bexiga, S. F. Nunes, C. Carneiro, L. M. Cavaco, F. Bernardo, and C. L. Vilela. 2006. Biofilm-forming ability profiling of Staphylococcus aureus and Staphylococcus epidermidis mastitis isolates. Vet. Microbiol. 118:133-140.

Oliveira, M., S. F. Nunes, C. Carneiro, R. Bexiga, F. Bernardo, and C. L. Vilela. 2007. Time course of biofilm formation by Staphylococcus aureus and Staphylococcus epidermidis mastitis isolates. Vet. Microbiol. 124:187-191.

Pereira, E. M., R. P. Schuenck, K. L. Malvar, N. L. Lorio, P. D. Matos, A. N. Olendzki, W. M. Oelemann, and K. R. Santos. 2010. Staphylococcus aureus, Staphylococcus epidermidis and Staphylococcus haemolyticus: Methicillin-resistant isolates are detected directly in blood cultures by multiplex PCR. Microbiol. Res. 165:243-249.

Pereira, V., C. Lopesa, A. Castroa, J. Silvaa, P. Gibbsa, and P. Teixeira. 2009. Characterization for enterotoxin production, virulence factors, and antibiotic susceptibility of Staphylococcus aureus isolates from various foods in Portugal. Food Microbiol. 26:278-282. 
Piessens, V., E. Van Coillie, B. Verbist, K. Supré, G. Braem, A. Van Nuffel, L. De Vuyst, M. Heyndrickx, and S. De Vliegher. 2011. Distribution of coagulase-negative Staphylococcus species from milk and environment of dairy cows differs between herds. J. Dairy Sci. 94:2933-2944.

Pitcher, D. G., N. A. Saunders, and R. J. Owen. 1989. Rapid extraction of bacterial genomic DNA with guanidium thiocyanate. Lett. Appl. Microbiol. 8:151-156.

Pyörälä, S. 2006. Treatment of clinical mastitis: Local and/or systemic? Short or long? Pages 250-259 in Proc. XIV World Buiat. Congr., Nice, France.

Pyörälä, S., and S. Taponen. 2009. Coagulase-negative staphylococciEmerging mastitis pathogens. Vet. Microbiol. 134:3-8.

Sakoulas, G., and R. Moellering Jr.. 2008. Increasing antibiotic resistance among methicillin resistant Staphylococcus aureus strains. Clin. Infect. Dis. 46(Suppl. 5):S360-367.

Semedo, T., M. A. Santos, M. F. Lopes, J. J. Figueiredo Marques, M. T. Barreto Crespo, and R. Tenreiro. 2003. Virulence factors in food, clinical and reference enterococci: A common trait in the genus? Syst. Appl. Microbiol. 26:13-22.

Sperber, W. H., and S. Tatini. 1975. Interpretation of the tube coagulase test for identification of Staphylococcus aureus. Appl. Microbiol. 29:502-505.

Tremblay, Y. D. N., D. Lamarche, P. Chever, D. Haine, S. Messier, and M. Jacques. 2013. Characterization of the ability of coagulase- negative staphylococci isolated from the milk of Canadian farms to form biofilms. J. Dairy Sci. 96:234-246.

Turkyilmaz, S., and O. Kaya. 2006. Determination of some virulence factors in Staphylococcus spp. isolated from various clinical samples. Turk. J. Vet. Anim. Sci. 30:127-132.

Vancraeynest, D., K. Hermans, and F. Haesebrouck. 2004. Genotypic and phenotypic screening of high and low virulence Staphylococcus aureus isolates from rabbits for biofilm formation and MSCRAMMs. Vet. Microbiol. 103:241-247.

Vasudevan, P., M. Nair, T. Annamalai, and K. Venkitanarayanan. 2003. Phenotypic and genotypic characterization of bovine mastitis isolates of Staphylococcus aureus for biofilm formation. Vet. Microbiol. 92:179-185.

Vautor, E., G. Abadie, A. Pont, and R. Thiery. 2008. Evaluation of the presence of the bap gene in Staphylococcus aureus isolates recovered from human and animals species. Vet. Microbiol. 127:407-411.

Virdis, S., C. Scarano, F. Cossu, V. Spanu, C. Spanu, and E. P. De Santis. 2010. Antibiotic resistance in Staphylococcus aureus and coagulase negative staphylococci isolated from goats with subclinical mastitis. Vet. Med. Int. 2010:517060. http://dx.doi. org/10.4061/2010/517060.

Walther, C., and V. Perreten. 2007. Methicillin-resistant Staphylococcus epidermidis in organic milk production. J. Dairy Sci. 90:5351.

Weckman, B. G., and B. W. Catlin. 1957. Deoxyribonuclease activity of micrococci from clinical sources. J. Bacteriol. 73:747-753. 